\title{
Feasibility Study Of Neutron Wavelength Compressor For EDM Measurement
}

\author{
Masaaki Kitaguchi* \\ Center for Experimental Studies, Nagoya University \\ E-mail: kitaguchi@phi.phys.nagoya-u.ac.jp
}

Yoshihisa Iwashita

Institute for Chemical Research, Kyoto University

\section{Hirohiko M. Shimizu}

Department of Physics, Nagoya University

\begin{abstract}
Neutrons can be accelerate and/or decelerate by using spin flip in the magnetic field. Controlling the number of spin-flips in the series of flippers enables us to change the energy of the acceleration/deceleration. The distribution of velocity of these neutrons can be concentrated by accelerating properly in the middle of the transport. The width of wavelength of the neutrons is compressed to improve the production of ultra-cold neutrons by superthermal method by using superfluid He-II. This has the advantage to improve the sensitivity of neutron EDM search.
\end{abstract}

The 26th International Nuclear Physics Conference

11-16 September, 2016

Adelaide, Australia

\footnotetext{
* Speaker.
} 


\section{Introduction}

The permanent electric dipole moment of neutrons (nEDM) signals the violation of timereversal invariance. Although experimental searches have been pursued in the world, the NEDM has not yet been observed. The present upper limit of nEDM is $\left|d_{n}\right|<3.0 \times 10^{-26} e \cdot \mathrm{cm}(90 \%$ C.L.), which is very close to the predictions of some physics beyond the standard model of particle physics, for example, supersymmetry [1]. For improvement of experimental sensitivity, the density of the stored ultra-cold neutrons (UCNs) is quite important in order to reduce the systematic errors from the uncertainty of the environment. One of the way to make dense UCNs is the superthermal UCN production [2] at accelerator-based spallation neutron source. The UCN sources are in operation at the Los Alamos National Laboratory (LANL) [3] and at the Paul Scherrer Institute (PSI) [4], under construction at TRIUMF based on the research at the Research Center for Nuclear Physics of Osaka University (RCNP) [5], and planned at the Spallation Neutron Source (SNS) [6]. The UCN converters at LANL and PSI are the solid ortho-deuterium (sD2). On the other hand, one of the most promising candidates of the converter at TRIUMF, RCNP, and SNS is superfluid helium-II at low temperature. The dispersion curve of a free neutron crosses that of helium twice. Coherent inelastic scattering provides a two energy level system for UCNs. The cold neutron with the velocity of $447 \mathrm{~m} / \mathrm{s}$ (the wavelength of $0.89 \mathrm{~nm}$ ), which corresponds to the crossing point of the dispersion curves, loses the kinetic energy to convert into UCNs directly by a scattering with one phonon in the converter. The cross section of the one phonon exchange process has a very sharp peak in incident neutron wavelength. Therefore, high intensity neutrons only at the specific region of the wavelength are requested to generate dense UCNs efficiently.

\section{Velocity concentration with spin flippers}

We are now discussing a new technique to get condensed neutrons at a specific wavelength. In the case of a pulsed neutron source, the bunched neutrons, which are generated instantaneously, spread spatially and temporally during its transport because of their velocity distribution. When fast neutrons are decelerated and/or slow neutrons are accelerated properly in the middle of the transport, the velocity of the neutrons can be controlled.

For the acceleration or deceleration of neutrons, we can utilize flipping of neutron spin in the magnetic field. The neutron polarity under a magnetic field $\boldsymbol{B}$ can be flipped by applying an rffield with the frequency of $v=2|\mu \boldsymbol{B}| / h$, where $\mu$ is the neutron magnetic moment. If the incident neutron spin polarity is positive, the relation between the kinetic energy of the incident neutron $\left(E_{\text {in }}\right)$ and that of the exit neutron $\left(E_{\mathrm{ex}}\right)$ is $E_{\mathrm{ex}}=E_{\text {in }}-h v$, and the neutron is decelerated by $h v$. On the other hand, negative polarity neutron is accelerated by $h v$. Here we consider the case where a main rf-cavity is installed in a strong-field region on the neutron beam path and an auxiliary rfcavity in a weak-field region as shown in Fig. 1. We refer to this device as a "spin flipper unit" below. The spin polarity is flipped to the original polarity in the auxiliary rf-cavity.

Figure 2 illustrates a series of identical spin flipper units placed with an equal spacing to which we refer as the "neutron velocity concentrator" [7]. When each rf-power is applied for a certain duration time, the number of deceleration or acceleration can be controlled according to time of flight of neutrons. Finally the neutrons have a same "target velocity". 


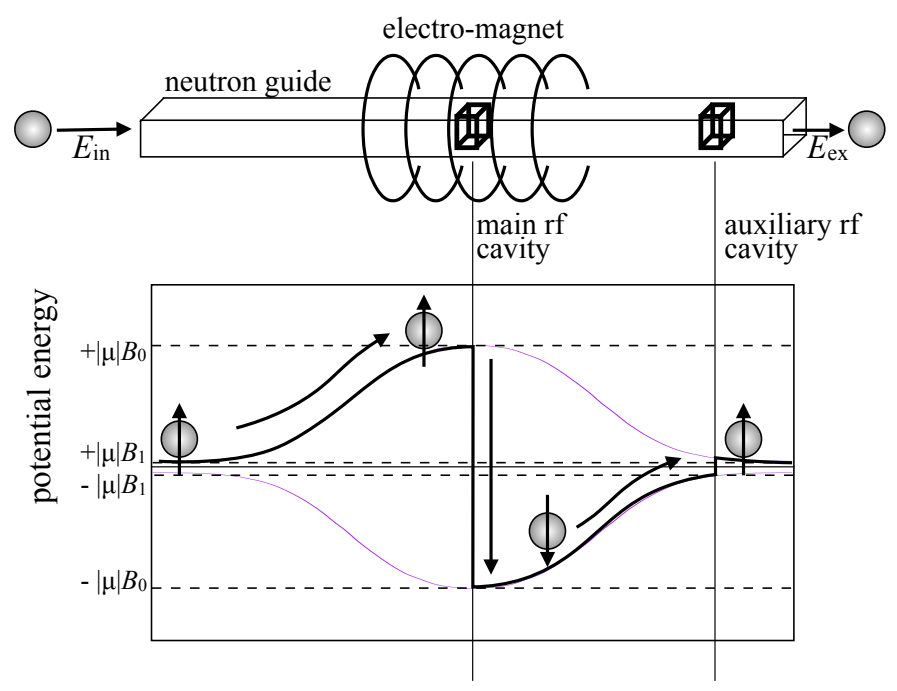

Figure 1: Schematic view of a spin flipper unit. A neutron with positive polarity is decelerated.

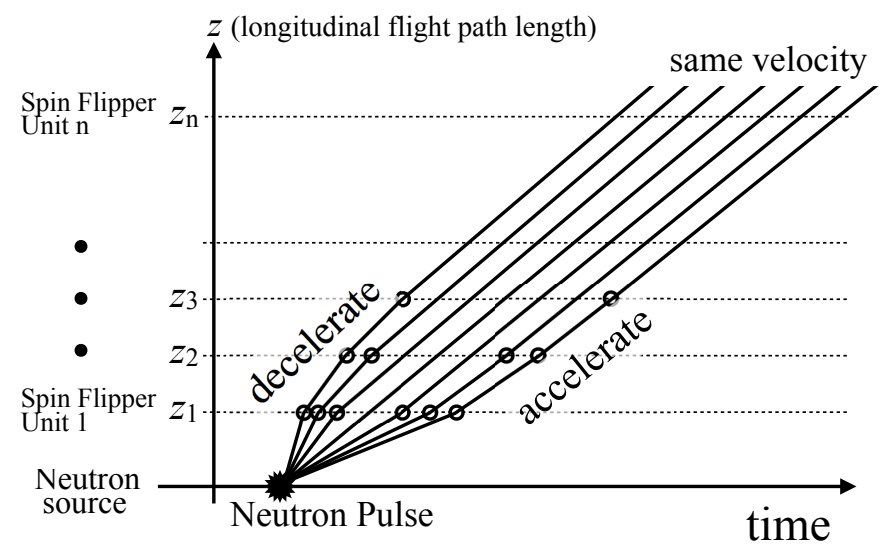

Figure 2: Schematic drawing of the neutron velocity concentrator.

\section{A design of neutron velocity concentrator for superthermal ultracold source with superfluid helium}

Now we consider sixty spin flipper units in the beamline (figure 3). The transport of pulsed neutrons are simulated by using Monte-Calro method. The target velocity of the velocity concentration was set to $447 \mathrm{~m} / \mathrm{s}$, which corresponds to one phonon exchange process of the superfluid helium as UCN converter. For a simple discussion, the neutrons with flat distribution of velocity were generated on the source at exactly same time.

Figure 4 shows the result of the velocity concentration. The sharp peak at the target velocity of $447 \mathrm{~m} / \mathrm{s}$ can be seen. The neutrons with the velocities from $435 \mathrm{~m} / \mathrm{s}$ to $447 \mathrm{~m} / \mathrm{s}$ were accelerated so that they have the same velocity of $447 \mathrm{~m} / \mathrm{s}$, and the neutrons with the velocities from $447 \mathrm{~m} / \mathrm{s}$ to $458 \mathrm{~m} / \mathrm{s}$ were decelerated so that they have the same velocity of $447 \mathrm{~m} / \mathrm{s}$. The full width of half maximum of the peak is $0.19 \mathrm{~m} / \mathrm{s}$, which corresponds to $0.85 \mu \mathrm{eV}$ of the change of energy by one flipper unit. The neutrons with the velocities slower than $435 \mathrm{~m} / \mathrm{s}$ were only accelerated by all of 


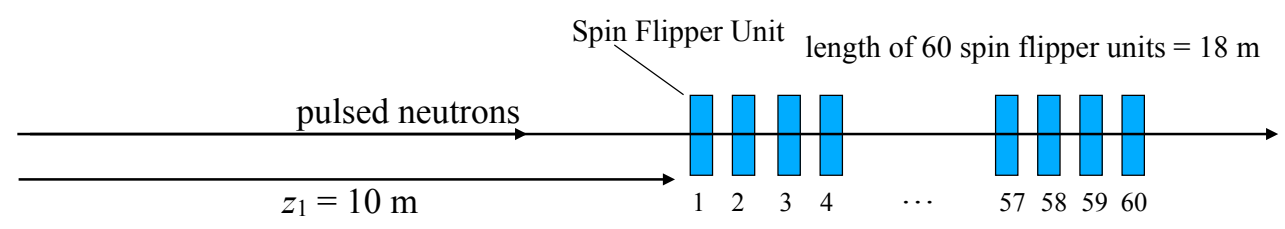

Figure 3: Beamline setup for simulation. Sixty flipper units were set on the beamline at the position of 10 $\mathrm{m}$ from the source.

the flipper units, and the neutrons with the velocities faster than $458 \mathrm{~m} / \mathrm{s}$ were only decelerated by all of the flipper units. The intensity around the target velocity increased by the order of 100 times with the manipulation.
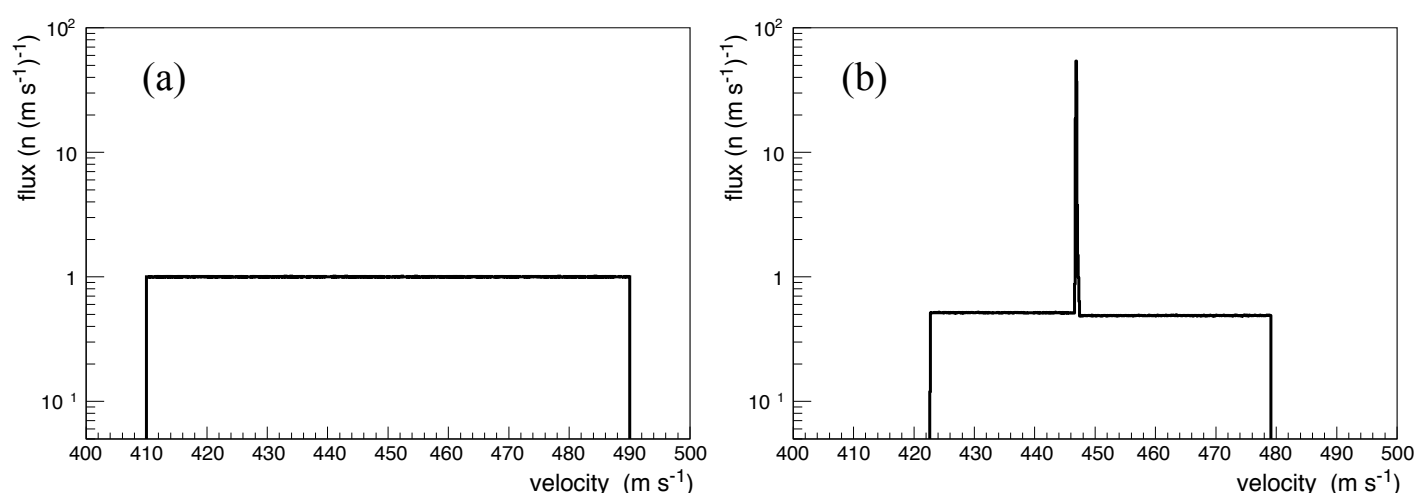

Figure 4: Result of simulation of the velocity concentration. (a) velocity distribution of flux of incident neutrons. (b) exit flux of preferred spin component.

\section{Conclusion}

The feasibility of neutron velocity concentration is demonstrated by using numerical simulations. In the case of pulsed neutrons, the intensity for the specific region of velocity increases by proper acceleration and/or deceleration for the surrounding velocity region of neutrons. This provides the more efficient production of UCNs by using the superthermal method with superfluid helium at low temperature. A possible enhancement with the velocity width of $0.19 \mathrm{~m} \mathrm{~s}^{-1}$ was estimated to be as large as 100 .

This technique can be applied to the nEDM measurement in the superfluid helium UCN converter located at the end of the neutron guide, for example, the fundamental physics beamline at the SNS. Even small amount of the spin flipper units gives us a gain of UCN production, which enables us to construst the beamline step by step. We emphasize that the controlling of neutron energy with high frequency spin flipper was already demonstrated by us [8] and the fast switching of RF flippers synchronizing with time-of-flight of neutron pulse is in operation stably in J-PARC [9]. These achievements indicate that the neutron velocity concentration can be realized by using the present techniques. 


\section{References}

[1] C.A. Baker, et al., Phys. Rev. Lett. 97131801 (2006).

[2] R. Golub, D.J. Richardson, S.K. Lamoreaux, Ultracold Neutrons, Adam Hilger, Bristol, 1991.

[3] A. Saunders, et al., Phys. Lett. B 59355 (2004).

[4] A. Anghel, et al., Nucl. Instr. and Meth. A 611272 (2009).

[5] Y. Masuda, et al., Phys. Rev. Lett. 89284801 (2002).

[6] N. Fomin, et. al., Nucl. Instr. and Meth. A 773, 45-51 (2015).

[7] M. Kitaguchi, et. al., arXiv 1611.05864 (2016).

[8] Y. Arimoto, et al., Phys. Rev., A 86, 023843 (2012).

[9] K. Taketani, et. al., Nucl. Instr. and Meth. A 634134 (2011). 